\title{
AVALIAÇÃO DA EFICIÊNCIA DOS REGIMES PRÓPRIOS DE PREVIDÊNCIA SOCIAL (RPPS) A PARTIR DA METODOLOGIA DEA
}

\author{
Luana Borges Sousa* \\ Adriano Firmino V. Araújo
}

\section{RESUMO}

Esse estudo objetiva avaliar a eficiência dos Regimes Próprios de Previdência Social (RPPS) para os estados e capitais estaduais no ano de 2014. Para tanto, foi utilizado o Modelo de Envoltória de Dados (DEA). Constatou-se que os RPPS dos estados do Distrito Federal e Amazonas são os dois mais eficientes, independentemente do tipo de retorno de escala que se considere. No caso das capitais, Natal-RN configura na primeira posição e São Luís-MA configura na última posição, independentemente do tipo de retorno de escala considerado.

Palavras-chaves: Eficiência, Regime Próprio de Previdência Social, Análise Envoltória de Dados.

\begin{abstract}
This study aims to evaluate the efficiency of Regimes of Public Employees called Regimes of Social Security (RPPS) for states and state capitals in the year 2014. To this end, we used the data envelopment analysis (DEA). It was found that the RPPS of the states of Amazonas and Federal District are the two most efficient, regardless of the scale of return type considered. In the case of capitals, NatalRN sets in the first position and São Luís-MA sets in last position, regardless of the type of return considered scale.
\end{abstract}

Keywords: Efficiency, Self Social Security System, Data envelopment analysis.

J EL: H55, C14

\footnotetext{
* Universidade Federal do Tocantins
} 


\section{IINTRODUÇÃO}

A previdência social é um seguro social que teve sua estruturação consolidada em 1988, com a promulgação da atual Constituição Federal. A Carta Magna veio consagrar o termo seguridade social no Brasil, definindo-o como um conjunto integrado de ações de iniciativa dos Poderes Públicos e da sociedade, destinado a assegurar os direitos relativos à saúde, à previdência e à assistência social (BRASIL, 1988).

Além de ser crucial para o bem-estar das pessoas e das famílias, a previdência social é fundamental para o funcionamento da economia e da sociedade como um todo. Segundo Scherman (2000, apud Thompson, 2000), além possibilitar a dignidade e a independência para seus beneficiários, permite, também, a manutenção da capacidade de consumo através dos benefícios em dinheiro. No entanto, nas últimas duas décadas a questão previdenciária tornou-se uma das grandes preocupações dos governos do mundo inteiro, pois os esquemas de financiamento que dão, ou deveriam dar, sustentação financeira e atuarial aos sistemas previdenciários entraram em crise.

Nesse sentido, é importante salientar a relação da crise previdenciária pública com o desenvolvimento da economia e a sociedade. Com o aumento da longevidade cumulado com o baixo índice de natalidade reserva aos cofres públicos a diminuição da arrecadação decorrente da subtração do número de segurados (financiadores) e o aumento do número e do tempo de concessão dos benefícios. Porém, entre as causas dessa situação, não há só o aumento da longevidade da população e a queda da taxa de natalidade, há, ainda, os rombos, o desvio de recursos, gestão ineficiente, além de, no caso da Previdência dos servidores públicos, a criação de certos benefícios para categorias específicas sem a devida fonte de custeio e as políticas paternalistas (Souza \& Reis, 2003).

No Brasil, o financiamento da previdência social é composto das receitas de recursos provenientes da União, dos Estados, do Distrito Federal, dos Municípios e de contribuições sociais. Os sistemas previdenciários existentes podem ser divididos em três: 1) Regime Geral de Previdência Social (RGPS) 2) Regime de Previdência Complementar (RPC) e 3) Regime Próprio dos Servidores Públicos denominado Regime Próprio de Previdência Social (RPPS).

Dentre os sistemas previdenciários enumerados há uma maior preocupação com o sistema previdenciário do servidor público devido ao aumento significativo no número de servidores inativos, impactando as despesas de pessoal dos entes federados nos últimos anos, o que representa uma transferência de recursos de toda a sociedade para essa parcela da população (Lewgoy, 2008).

Desse modo, diante da grave crise da previdência social no Brasil (Giambiagi, 2014), tendo como consequência o déficit atuarial devido, principalmente, ao envelhecimento da população, pois há, cada vez mais, menos contribuintes por beneficiário (Follador, 2014). A eficiência dos RPPS é necessária para alcançar a meta da previdência social que é a proteção, pagamento das aposentadorias e pensões, dos segurados. Os RPPS possuem recursos como as contribuições repassadas do Ente, dos servidores, dos aposentados e dos pensionistas, além 
das transferências, aportes, contribuições arrecadadas dos militares e as contribuições arrecadadas pela unidade gestora, entre outros. No entanto, há também saídas de recursos, como as aposentadorias, as pensões, as despesas administrativas e as demais utilizações de recursos.

Nesse contexto, o presente estudo propõe a seguinte problematização: Quais RPPS estaduais e de capitais estaduais são eficientes quanto à evolução patrimonial no ano de 2014, dadas suas entradas e saídas de recursos?

Diante desta problematização, o objetivo geral da dissertação é avaliar, primeiramente, os RPPS estaduais e depois os RPPS de capitais estaduais quanto à sua eficiência relativa na evolução patrimonial a partir da metodologia DEA.

O presente estudo está dividido em cinco capítulos, sendo este o primeiro. O segundo aborda os aspectos conceituais e técnicos da previdência social, com ênfase no RPPS. A metodologia é o terceiro capítulo, que trata da análise de eficiência e do método aplicado, o quarto capítulo expõe os resultados e as discussões dos mesmos, as considerações finais é o quinto capítulo.

2 PREVIDÊNCIA SOCIAL

\subsection{Seguridade Social e Previdência}

A previdência social é garantida pela seguridade social ou, ainda, segurança social. A Constituição Federal de 1988 define seguridade social:

Art. 194. A seguridade social compreende um conjunto integrado de ações de iniciativa dos Poderes Públicos e da sociedade, destinadas a assegurar os direitos relativos à saúde, à previdência e à assistência social (BRASIL, 1988).

De acordo com Boschetti (2003) o conceito de Seguridade Social tem como parâmetro o Modelo clássico de William Henry Beveridge que, em 1942, estabeleceu o divisor entre seguro e os avanços sociais, determinando a universalização dos direitos sociais destinados a todos os cidadãos, incondicionalmente ou submetidos a condicionantes, mas garantindo o mínimo a todos. Portanto, a Seguridade Social é um sistema de proteção social que abrange os três programas sociais de maior relevância para população: a saúde, que é direito de todos e dever do Estado; a assistência social, que é prestada a quem dela necessitar, independentemente de contribuição à seguridade social; e pela previdência social, que se preocupa exclusivamente com os trabalhadores e com os seus dependentes econômicos.

A Constituição Federal de 1988, art. 6º estabelece a previdência social como um direito social. 
Art. 6o São direitos sociais a educação, a saúde, a alimentação, o trabalho, a moradia, o lazer, a segurança, a previdência social, a proteção à maternidade e à infância, a assistência aos desamparados, na forma desta Constituição (BRASIL, 1988).

Cabe ressaltar que, no Brasil, os direitos sociais são considerados cláusulas pétreas, protegidos pela imutabilidade. Ou seja, não é possível a supressão dos direitos fundamentais sociais. Mais especificamente, não é possível a supressão do direito à Previdência Social. Balera (2003) afirma que a previdência social pode ser definida como uma poupança forçada, imposta ao cidadão para que este possua condições financeiras de usufruir da vida em sociedade quando não mais possuir capacidade laboral. Diante destas definições, o presente estudo trabalha a previdência social como um seguro social que o trabalhador tem o direito de usufruir, quando é atingido por um dos chamados riscos sociais: doença, invalidez, idade avançada, morte, desemprego involuntário, maternidade e a reclusão.

\subsection{Regimes Previdenciários}

Os regimes previdenciários englobam os indivíduos que possuem vinculação em virtude de relações de trabalho ou categoria profissional. Para esses indivíduos são garantidos, no mínimo, os benefícios observados em todo sistema de seguro social. As principais formas de regime previdenciário são: 0 Regime Geral de Previdência Social (RGPS), Regime de Previdência Complementar (RPC) e o Regime Próprio de Previdência Social (RPPS). Este, que por sua vez é o objeto de estudo, apresenta uma discussão mais aprofundada.

\subsubsection{Regime Geral de Previdência Social (RGPS)}

O Regime Geral de Previdência Social é o principal regime previdenciário no Ordenamento J urídico Brasileiro, abarcando, de forma obrigatória, todos os trabalhadores da iniciativa privada, ou seja, os que possuem relação de emprego regida pela Consolidação das Leis do Trabalho (CLT), bem assim os empregados rurais, domésticos, trabalhadores autônomos, empresários e trabalhadores avulsos. Além dos trabalhadores acima mencionados, o Regime Geral de Previdência Social (RGPS) contempla também alguns servidores públicos efetivos, desde que os mesmos não se encontrem amparados pelo Regime Próprio de Previdência Social (RPSS), e que haja o exercício de atividade remunerada.

No Brasil, qualquer pessoa, nacional ou não, que venha a exercer atividade remunerada em território brasileiro filia-se, automaticamente, ao Regime Geral de Previdência Social - RGPS, 
sendo obrigada a efetuar recolhimentos ao sistema previdenciário (somente se excluem desta regra as pessoas já vinculadas a regimes próprios de previdência) (Ibrahim, 2005 : 21).

O Ministério da Previdência Social (MPS) é o órgão responsável pela administração do RGPS. É o MPS que elabora suas políticas, estas que são executadas pelo Instituto Nacional do Seguro Social (INSS), autarquia federal a ele vinculado.

\subsubsection{Regime de Previdência Complementar (RPC)}

A Previdência Complementar Privada foi instituída pela Lei $n^{\circ}$ 6.435/77. No entanto, esta lei foi revogada em 29 de maio de 2001 pela Lei Complementar $n^{\circ}$ 109 que estabelece o regime de previdência privada como de caráter complementar e organizado de forma autônoma em relação ao Regime Geral de Previdência Social (RGPS). Além disso o regime é facultativo e baseado na constituição de reservas que garantam o benefício, nos termos do caput do art. 202 da CF/ 88.

O Regime de Previdência Complementar (RPC) tem suas políticas elaboradas pelo Ministério da Previdência Social (MPS) e executadas pela Superintendência Nacional de Previdência Complementar (PREVIC).

No Brasil o RPC é organizado em dois segmentos: o segmento operado pelas entidades abertas - com acesso individual, e o segmento operado pelas Entidades Fechadas de Previdência Complementar (EFPCs), também conhecidas como fundos de pensão, que operam Planos de Benefícios destinados aos empregados de empresa ou grupo destas, denominadas patrocinadoras, bem como aos associados ou membros de associações, entidades de caráter profissional, classista ou setorial, denominados de instituidores (MPS, 2015).

\section{2.3 Regimes Próprios de Previdência Social (RPPS)}

O Regime Próprio de Previdência Social (RPPS) é o regime de previdência assegurado exclusivamente aos servidores públicos titulares de cargo efetivo, mantido pelos entes públicos da Federação (União, Estados, Distrito Federal e Municípios). Este regime tem suas políticas elaboradas e executadas pelo Ministério da Previdência Social (MPS).

A Lei $n^{\circ}$ 9.717, de 27 de novembro de 1998, conhecida como a Lei Geral de Previdência Pública dispôs sobre as regras gerais para a organização e o funcionamento dos Regimes Próprios de Previdência Social (RPPS) dos Servidores Públicos da União, dos Estados, do Distrito Federal e dos Municípios e os militares dos Estados e do Distrito Federal. Esta lei foi modificada pela EC $\mathrm{n}^{\circ} 20 / 98$ : 
Art. 201. A previdência social será organizada sob a forma de regime geral, de caráter contributivo e de filiação obrigatória, observados critérios que preservem o equilíbrio financeiro e atuarial, e atenderá, nos termos da lei, a: I - cobertura dos eventos de doença, invalidez, morte e idade avançada; II - proteção à maternidade, especialmente à gestante; III - proteção ao trabalhador em situação de desemprego involuntário; IV - salário-família e auxílio-reclusão para os dependentes dos segurados de baixa renda; V - pensão por morte do segurado, homem ou mulher, ao cônjuge ou companheiro edependentes (EC $\left.{ }^{\circ} 20 / 98\right)$.

Nesse sentido, com o objetivo de fazer com que os Regimes Próprios de Previdência Social (RPSS) seguissem as normas de boa gestão, de forma a assegurar o pagamento dos benefícios previdenciários aos seus segurados, foi instituído no âmbito da administração pública federal, o Certificado de Regularidade Previdenciária (CRP), documento é fornecido pela Secretaria de Políticas de Previdência Social (SPS), do Ministério da Previdência Social.

Quanto aos recursos dos RPPS, segundo o Ministério da Previdência Social (MPS), estes são considerados recursos previdenciários as disponibilidades oriundas das receitas correntes e de capital, as aplicações financeiras, os títulos e os valores mobiliários, os demais ingressos financeiros auferidos pelo RPPS, os ativos vinculados por lei ao RPPS e demais bens, direitos e ativos com finalidade previdenciária do RPPS.

O equilíbrio financeiro e atuarial dos Regimes Próprios de Previdência Social (RPSS) é exigido pela $\mathrm{CF} / 88$ :

Art. 40. Aos servidores titulares de cargos efetivos da União, dos Estados, do Distrito Federal e dos Municípios, incluídas suas autarquias e fundações, é assegurado o regime de previdência de caráter contributivo e solidário, mediante contribuição do respectivo ente público, dos servidores ativos e inativos e dos pensionistas, observados critérios que preservem o equilíbrio financeiro e atuarial e o disposto neste artigo. (BRASIL, 1988).

\section{METODOLOGIA}

As informações utilizadas neste estudo foram obtidas junto ao Sistema de Informações dos Regimes Próprio de Previdência Social do Ministério da Previdência Social (CADPREV). Consistem em informações relacionadas aos Regimes Próprios de Previdência Social (RPPS) estaduais e das capitais estaduais, os quais consistem nos maiores RPPS brasileiros. O ano de 2014 foi escolhido por apresentar informações para maior número de estados e capitais estaduais.

As informações utilizadas se referem às receitas previdenciárias, número de beneficiários, despesas previdenciárias, total de benefícios e incremento do patrimônio líquido. As receitas, número de beneficiários, as despesas e o total de benefícios serão retirados do Demonstrativo de Informações Previdenciárias 
e Repasses (DIPR). Quanto ao patrimônio líquido, será retirado do Demonstrativo das Aplicações e Investimentos dos Recursos (DAIR).

Como dito anteriormente, nem todos os estados e capitais estaduais apresentaram todos os dados para o ano de 2014. Especialmente, os estados do Amapá, Mato Grosso e Paraná e as capitais estaduais Brasília, Maceió, Porto Velho e Rio de J aneiro não foram analisados por falta de informação. O quadro 01 apresenta os estados e capitais analisados.

QUADRO 01 - ESTADOS E CAPITAIS ESTADUAIS COM SUAS DMUS RESPECTIVAS.

\begin{tabular}{|c|c|c|c|}
\hline ESTADOS & DMU & CAPITAIS ESTADUAIS & DMU \\
\hline ACRE & AC & ARACAJU & ARA \\
ALAGOAS & AL & BELÉM & BEL \\
AMAZONAS & AM & BELO HORIZONTE & BH \\
BAHIA & BA & BOA VISTA & BV \\
CEARÁ & CE & CAMPO GRANDE & CG \\
DISTRITO FEDERAL & DF & CUIABÁ & CUI \\
ESPÍRITO SANTO & ES & CURITIBA & CUR \\
GOIÁS & GO & FLORIANÓPOLIS & FLO \\
MARANHÃO & MA & FORTALEZA & FOR \\
MATO GROSSO DO SUL & MS & GOIÂNIA & GOI \\
MINAS GERAIS & MG & JOÃO PESSOA & JP \\
PARÁ & PA & MACAPÁ & MAC \\
PARAÍBA & PB & MANAUS & MAN \\
PERNAMBUCO & PE & NATAL & NAT \\
PIAUI & PI & PALMAS & PAL \\
RIO DE JANEIRO & RJ & PORTO ALEGRE & POA \\
RIO GRANDE DO NORTE & RN & RECIFE & REC \\
RIO GRANDE DO SUL & RS & RIO BRANCO & RB \\
RONDÔNIA & RO & SALVADOR & SAL \\
RORAIMA & RR & SÃO LUÍS & SL \\
SANTA CATARINA & SC & SÃO PAULO & SP \\
SÃO PAULO & SP & TEREZINHA & TER \\
SERGIPE & SE & VITÓRIA & VIT \\
TOCANTINS & TO & & \\
& & &
\end{tabular}

Fonte: Elaborado pelos autores 


\subsection{Análise Envoltória dos Dados (DEA)}

A Análise Envoltória de Dados (Data Envelopment Analysis - DEA) é um método de programação linear não paramétrico de medida de eficiência (Soares de Mello et al, 2003). Baseada fundamentalmente nos trabalhos conduzidos por Farrel (1957), que foi mais amplamente discutido e aprofundado por Charnes et al. (1978), e Banker et al. (1984). (Lins \& Ângulo Meza, 2000). Pode ser definida, ainda, por uma ferramenta não-paramétrica que avalia a eficiência técnica relativa de unidades produtivas, chamadas de Unidades tomadoras de decisão (DMU - Decision Making Units), comparando entidades que realizam tarefas similares e se diferenciam pela quantidade de recursos utilizados (inputs) e de bens produzidos (outputs).

Segundo Cooper; Seiford e Tone (2000), os elementos básicos de uma aplicação DEA são os seguintes: a) Decision Making Unit (DMU) ou unidade tomadora de decisão: trata-se da unidade produtiva que se deseja avaliar e comparar com outras unidades da mesma natureza, sendo esta responsável pela conversão de entradas em saídas; b) inputs ou entradas são os insumos, como matéria prima, equipamento, capital, horas de trabalho, energia e tempo empregados pela DMU na geração de uma determinada produção; c) outputs ou saídas são os produtos gerados pela DMU como bens ou serviços produzidos ou vendidos; uma DMU pode ter uma ou mais saídas; d) modelo escolhido: DEA permite a escolha de vários modelos de cálculos segundo a sua adequação, como o CCR (Charnes-Cooper-Rhodes) e BCC (Banker-Charnes-Cooper), com orientação à entrada (ou inputs) ou a saídas (ou outputs); e) fronteira de eficiência, que é construída a partir dos melhores resultados apresentados pelo conjunto de DMUs, para essas DMUs é atribuído o valor máximo de eficiência (1 ou 100\%); f) eficiência relativa, refere-se ao valor de eficiência (ou ineficiência) das DMUs em relação à fronteira; e g) pesos calculados: os melhores pesos para cada DMU de cada entrada e saída são atribuídos, visando atingir a maior eficiência possível.

Quando muitas DMUs conseguem o score de 100\% de eficiência evidencia uma limitação da fronteira clássica da DEA em discriminar as unidades eficientes. Diante disso, com o objetivo de melhor discriminar as DMUs, Yamada et al. (1994), Entani et al. (2002) e Novaes (2002) apud Soares de Mello et al. (2003), desenvolveram o conceito de fronteira invertida.

A fronteira invertida consiste em considerar os outputs como inputs e os inputs como outputs (Novaes, 2002). A fronteira invertida é utilizada na construção de um índice chamado de índice de eficiência composta. Ele é definido como sendo a média aritmética entre a eficiência em relação à fronteira DEA convencional (padrão) e o complemento da eficiência em relação à fronteira invertida. O uso do complemento justifica-se pelo fato de a fronteira invertida gerar uma medida de ineficiência.

Uma maneira fazer um ranking entre as DMUs é calculando a eficiência composta normalizada, pois esta é única para cada DMU. A eficiência composta normalizada é obtida dividindo o valor da eficiência composta pelo maior valor entre todos os valores de eficiência composta (Ângulo Meza et al., 2005). 
Nesse sentido, a abordagem DEA admite dois tipos de aplicação, cada um com dois tipos de orientação: CCR - insumo orientado, CCR - produto orientado, BCC - insumo orientado e BCC - produto orientado. Esses quatro modelos estão detalhados em Fried et al. (1993) e Charnes et al. (1994). Portanto, deve-se fazer uma opção, o modelo orientado a outputs obtém o máximo nível de outputs mantendo os inputs fixos, ou um modelo orientado a inputs, que visa a obter um menor uso de inputs dado o nível dos outputs. A decisão de usar um ou outro modelo deve ser previamente selecionada pelo pesquisador.

Banker, Charnes e Cooper (1984) definiram uma regra baseada em programação matemática que determina o número de inputs e outputs que podem ser utilizados com relação à quantidade de DMUs a serem analisadas. De acordo com esses autores, a soma de inputs e outputs deve ser menor ou igual a um terço da quantidade de DMU. Logo, foram consideradas cinco variáveis inputs/ outputs, conforme apresentado no quadro 02. Ressalta-se que se adotou as mesmas variáveis e mesma classificação inputs/outputs para avaliação dos RPPS estaduais e de capitais estaduais.

QUADRO 02- INSUMOS E PRODUTOS.

\begin{tabular}{|l|c|}
\hline \multicolumn{1}{|c|}{ DADOS } & CLASSIFICAÇÃO \\
\hline Receitas previdenciárias & INPUT 1 \\
Número de beneficiários & INPUT 2 \\
Despesas previdenciárias & INPUT 3 \\
Total de benefícios & OUTPUT 1 \\
Incremento do patrimônio líquido & OUTPUT 2 \\
\hline
\end{tabular}

Fonte: Elaborado pelos autores.

Desse modo, para a utilização da metodologia DEA, foi empregado, nessa avaliação, o modelo clássico de Retornos Variáveis de Escala (BCC), mais adequado, e o Retorno Constante de Escala, de modo que pudéssemos comparar os resultados. Quanto à orientação, esta foi para os produtos (outputs), pois os RPPS buscam um alto e sustentável patrimônio líquido, suficiente para pagamento dos benefícios (aposentadorias e pensões) atuais e futuros, dado as entradas de recursos do RPPS.

O objetivo desta pesquisa é avaliar a eficiência dos RPPS estaduais e de capitais estaduais, desse modo, faz-se uma avaliação dos RPPS estaduais e logo após trabalha-se com a avaliação dos RPPS das capitais estaduais. Nesse sentido, primeiramente, encontra-se a taxa de eficiência relativa dos RPPS estaduais, isto é, determina quão eficiente é um RPPS estadual ao transformar seus insumos (entradas de recursos e número de beneficiários) em produtos (pagamento de benefícios e incremento do patrimônio líquido), comparando aos demais RPSS estaduais observados (os escores de eficiência serão encontrados com a utilização do Sistema Integrado de Apoio à Decisão - SIAD). O mesmo processo é utilizado para comparar a eficiência dos RPPS de capitais estaduais. 
Os resultados completos do software SIAD fornecem a eficiência padrão, a eficiência usando fronteira invertida - que identifica os RPPS que inicialmente são apresentadas como eficientes, contudo podem representar uma falsa eficiência -, o índice composto (eficiência padrão e invertida), a eficiência composta normalizada - que é única para cada RPPS, o que possibilita fazer um ranking do RPPS mais eficiente até o menos eficiente - os pesos das variáveis (inputs e outputs) utilizadas no modelo, os benchmarks - que identifica os RPPS eficientes que servem de referência para os RPPS ineficientes - e os alvos para os RPPS - que incluem as folgas e os níveis que cada variável deve atingir para os RPPS serem eficientes.

4 RESULTADOS

\subsection{ANALISE DA EFICIÊNCIA DOS RPPS ESTADUAIS}

A tabela 03 mostra os valores de todos os inputs: receitas, número de beneficiários e despesas; e outputs: total de benefícios e incremento do patrimônio líquido.

TABELA 03 - INPUTS E OUTPUTS DOS RPPS ESTADUAIS.

\begin{tabular}{cccccc}
\hline DMU & Input1 & Input2 & Input3 & Output1 & Output2 \\
\hline AC & 479385170 & 8328 & 438968828 & 343009747 & 55553486 \\
AL & 1331657998 & 2537 & 1308067161 & 370109406 & 18037346 \\
AM & 1504929954 & 2713 & 1124503543 & 621444123 & 457569128 \\
BA & 5198161243 & 94643 & 4973339753 & 4974692632 & 362775500 \\
CE & 2427453760 & 50449 & 2414205404 & 1997203947 & 25664434 \\
DF & 3836671314 & 52074 & 1596633868 & 5154582448 & 866680533 \\
ES & 2489310901 & 28458 & 2067081401 & 1741246836 & 394575078 \\
GO & 3626656737 & 59560 & 2686330135 & 2655587080 & 14218597 \\
MA & 1001363674 & 3914 & 1351990801 & 543038050 & 53600447 \\
MS & 1715553538 & 21567 & 1684384845 & 1465311752 & 35856059 \\
MG & 8468988875 & 379629 & 7489476930 & 6485527807 & -2535036400 \\
PA & 3430723592 & 36998 & 2391868712 & 2021558280 & 727798842 \\
PB & 1660998353 & 36984 & 1611822011 & 1350781782 & 36485425 \\
PE & 3733435740 & 81774 & 3832350549 & 3817563592 & -96167595 \\
PI & 1278435516 & 32802 & 1383507091 & 1148023523 & -10020902 \\
RJ & 25112144991 & 169545 & 13986842511 & 10736770046 & -22522593842 \\
RN & 1076724110 & 35551 & 1015720542 & 1305159337 & 65945907 \\
RS & 10245617796 & 172512 & 9230578440 & 8024254505 & 154444620
\end{tabular}




\begin{tabular}{cccccc}
\hline DMU & Input1 & Input2 & Input3 & Output1 & Output2 \\
\hline RO & 547239700 & 5118 & 278800937 & 225427791 & 265913681 \\
RR & 4860885273 & 14 & 23733420 & 2901160 & 290279557 \\
SC & 4267318363 & 50410 & 4357169988 & 3418003025 & 179588268 \\
SP & 25416852099 & 314558 & 25474232824 & 18496703445 & 1669757 \\
SE & 1749683452 & 22784 & 1473178530 & 1211175758 & 187771217 \\
TO & 1246709863 & 8678 & 388670038 & 381654502 & 567018142 \\
\hline
\end{tabular}

Fonte: Elaborado pelos autores.

Após a inserção dos dados, foi escolhido, primeiramente, o modelo DEA/BCC orientação output, após dar o comando de cálculo e salvar os resultados, realizou-se o mesmo procedimento com o modelo DEA/CCR. Os resultados de eficiência padrão, invertida e eficiência composta normalizada estão expostas na tabela 04.

TABELA 04 - EFICIÊNCIAS DOS RPPS ESTADUAIS.

\begin{tabular}{ccccccc}
\hline \multirow{2}{*}{ DMU } & \multicolumn{3}{c}{ DEA/BCC } & & DEA/CCR & \\
\cline { 2 - 7 } & Padrão & Invertida & Composta* & Padrão & Invertida & Composta* \\
\hline AC & 1.000 .000 & 1.000 .000 & 0,556239 & 0,532578 & 0,621724 & 0,506282 \\
AL & 1.000 .000 & 1.000 .000 & 0,556239 & 0,660114 & 1.000 .000 & 0,366913 \\
AM & 1.000 .000 & 0,537951 & 0,813248 & 1.000 .000 & 0,518005 & 0,823741 \\
BA & 0,829622 & 0,672835 & 0,643450 & 0,712324 & 0,486891 & 0,681135 \\
CE & 0,626263 & 0,688216 & 0,521778 & 0,612396 & 0,664703 & 0,526758 \\
DF & 1.000 .000 & 0,202211 & 1.000 .000 & 1.000 .000 & 0,200896 & 1.000 .000 \\
ES & 0,618437 & 0,600664 & 0,566125 & 0,617933 & 0,502612 & 0,619932 \\
GO & 0,546230 & 0,762573 & 0,435900 & 0,545023 & 0,680098 & 0,480753 \\
MA & 1.000 .000 & 0,787900 & 0,674217 & 0,935902 & 0,732432 & 0,668928 \\
MS & 0,742125 & 0,494952 & 0,693726 & 0,675556 & 0,486693 & 0,660808 \\
MG & 0,808816 & 1.000 .000 & 0,449895 & 0,570000 & 1.000 .000 & 0,316824 \\
PA & 1.000 .000 & 0,676071 & 0,736420 & 0,648043 & 0,524114 & 0,624716 \\
PB & 0,640543 & 0,677706 & 0,535567 & 0,605309 & 0,637298 & 0,538052 \\
PE & 0,761898 & 1.000 .000 & 0,423797 & 0,761095 & 0,829416 & 0,517857 \\
PI & 0,733604 & 1.000 .000 & 0,408059 & 0,668395 & 0,960691 & 0,393365 \\
RJ & 1.000 .000 & 1.000 .000 & 0,556239 & 0,543335 & 1.000 .000 & 0,302003 \\
RN & 1.000 .000 & 1.000 .000 & 0,556239 & 0,902236 & 0,527632 & 0,764050 \\
RS & 0,880145 & 0,836137 & 0,580718 & 0,582945 & 0,573479 & 0,561094 \\
RO & 1.000 .000 & 0,754789 & 0,692635 & 1.000 .000 & 0,584740 & 0,786647 \\
RR & 1.000 .000 & 1.000 .000 & 0,556239 & 1.000 .000 & 1.000 .000 & 0,555832 \\
SC & 0,699267 & 0,803635 & 0,498185 & 0,665049 & 0,507353 & 0,643485 \\
SP & 1.000 .000 & 1.000 .000 & 0,556239 & 0,582706 & 1.000 .000 & 0,323887
\end{tabular}




\begin{tabular}{ccccccc}
\hline \multirow{2}{*}{ DMU } & \multicolumn{3}{c}{ DEA/BCC } & \multicolumn{3}{c}{ DEA/CCR } \\
\cline { 2 - 7 } & Padrão & Invertida & Composta* & Padrão & Invertida & Composta* \\
SE & 0,601269 & 0,561592 & 0,578309 & 0,532503 & 0,540065 & 0,551629 \\
TO & 1.000 .000 & 1.000 .000 & 0,556239 & 1.000 .000 & 0,527282 & 0,818584 \\
\hline
\end{tabular}

*Eficiência normalizada.

Fonte: Elaborado pelos autores.

Quanto à eficiência padrão, ao observar os resultados, percebe-se que enquanto o modelo DEA/BCC identificou doze estados eficientes e doze ineficientes, o modelo DEA/CCR identificou cinco estados eficientes e dezenove ineficientes. Os cinco RPPS estaduais eficientes no modelo DEA/CCR são também eficientes no modelo DEA/BCC.

Quanto à eficiência encontrada pela fronteira invertida, esta permite a identificação de RPPS considerados "falsos eficientes", pois RPPS considerados eficientes através da fronteira padrão são considerados ineficientes através da fronteira invertida, caracterizando uma falsa eficiência.

Portanto, todos os RPPS eficientes na fronteira padrão teoricamente passariam ao status de ineficientes na segunda fronteira e vice-versa. Como a DEA atribui um score específico para RPPS como pertencente à área de ineficiência então se teria uma maneira de desempatar as empresas consideradas 100\% eficientes pela fronteira padrão, não fosse pelo fato de alguns RPPS eficientes na primeira fronteira também serem considerados eficientes na segunda fronteira, como foi o caso, de acordo com a tabela 04, no modelo DEA/BCC, dos RPPS de Acre, Alagoas, Rio de J aneiro, Rio Grande do Norte, Roraima, São Paulo e Tocantins. No modelo DEA/ CCR somente o RPPS de Roraima apresentou "falsa eficiência" na fronteira invertida.

A explicação de o modelo BCC ter apresentado um grande número de RPPS estaduais eficientes, conforme tabela 05 , pode estar nas características matemáticas deste modelo, pois as DMUs que têm o menor valor de um dos inputs ou o maior valor de um dos outputs são consideradas eficientes, mesmo que as relações com as demais variáveis não sejam as melhores (Ali, 1993). Esses RPPS estaduais são chamados de falsamente eficientes.

Há vários métodos para melhorar a discriminação quando utiliza-se modelos DEA (Angulo Meza \& Lins, 2002; Leta et al., 2005). Um desses métodos, que tem a vantagem adicional de eliminar as DMUs falsamente eficientes no modelo BCC, é a fronteira invertida.

A partir da eficiência invertida é construída a eficiência composta. Esta é a média aritmética entre a eficiência em relação à fronteira DEA convencional (padrão) e o complemento da eficiência em relação à fronteira invertida. 
TABELA 05 - RPPS ESTADUAIS EFICIENTES E INEFICIENTES EFICIÊNCIA PADRÃO.

\begin{tabular}{llll}
\hline \multicolumn{2}{c}{ EFICIENTES } & \multicolumn{2}{c}{ INEFICIENTES } \\
\hline \multicolumn{1}{c}{ DEA/BCC } & DEA/CCR & \multicolumn{1}{c}{ DEA/BCC } & \multicolumn{1}{c}{ DEA/CCR } \\
\hline Acre & Amazonas & Bahia & Acre \\
Alagoas & Distrito F. & Ceará & Alagoas \\
Amazonas & Rondônia & Espirito Santo & Bahia \\
Distrito F. & Roraima & Goiás & Ceará \\
Maranhão & Tocantins & Mato Grosso do Sul & Espirito Santo \\
Pará & & Minas Gerais & Goiás \\
Rio deJ aneiro & & Paraíba & Maranhão \\
RN & & Pernambuco & Mato Grosso do Sul \\
Rondônia & & Piauí & Minas Gerais \\
Roraima & & Rio Grande do Sul & Pará \\
São Paulo & & Santa Catarina & Paraíba \\
Tocantins & & Sergipe & Pernambuco \\
& & & Piauí \\
& & & Rio deJaneiro \\
& & & Rio Grande do Norte \\
& & & Rio Grande do Sul \\
& & & Santa Catarina \\
& & & São Paulo \\
& & & Sergipe \\
\hline
\end{tabular}

Fonte: Elaborado pelos autores.

Ao encontrar o valor da eficiência composta e dividi-lo pelo maior valor entre todos os valores de eficiência composta encontra-se a eficiência composta normalizada. Esta eficiência é única para cada RPPS estadual, portanto, a partir dela faz-se um ranking, tabela 06, dos RPPS estaduais mais eficientes.

O RPPS do Distrito Federal é o mais eficiente, tanto no modelo DEA/BCC quanto no modelo DEA/CCR, o mesmo acontece com o segundo lugar, o RPPS de Amazonas. No entanto, a partir da terceira posição os modelos divergem nos resultados. Ressalta-se que as posições se divergem devido os dois modelos utilizados apresentarem retornos de escala diferentes, então o DEA/BCC considera todos os retornos, o DEA/CCR considera apenas o retorno constante de escala. 
TABELA 06 - RANKING DOS RPPS ESTADUAIS - EFICIÊNCIA NORMALIZADA.

\begin{tabular}{cll}
\hline Posição & \multicolumn{1}{c}{ DEA/BCC } & \multicolumn{1}{c}{ DEA/CCR } \\
\hline $1^{\underline{a}}$ & Distrito Federal & Distrito Federal \\
$2^{\underline{a}}$ & Amazonas & Amazonas \\
$3^{\underline{a}}$ & Pará & Tocantins \\
$4^{\underline{a}}$ & Mato Grosso do Sul & Rondônia \\
$5^{\underline{a}}$ & Rondônia & Rio Grande do Norte \\
$6^{\underline{a}}$ & Maranhão & Bahia \\
$7^{\underline{a}}$ & Bahia & Maranhão \\
$8^{\underline{a}}$ & Rio Grande do Sul & Mato Grosso do Sul \\
$9^{\underline{a}}$ & Sergipe & Santa Catarina \\
$10^{\underline{a}}$ & Espírito Santo & Pará \\
$11^{\underline{a}}$ & Acre & Espírito Santo \\
$12^{\underline{a}}$ & Alagoas & Rio Grande do Sul \\
$13^{\underline{a}}$ & Rio deJ Janeiro & Roraima \\
$14^{\underline{a}}$ & Rio Grande do Norte & Sergipe \\
$15^{\underline{a}}$ & Roraima & Paraíba \\
$16^{\underline{a}}$ & São Paulo & Ceará \\
$17^{\underline{a}}$ & Tocantins & Pernambuco \\
$18^{\underline{a}}$ & Paraíba & Acre \\
$19^{\underline{a}}$ & Ceará & Goiás \\
$20^{\underline{a}}$ & Santa Catarina & Piauí \\
$21^{\underline{a}}$ & Minas Gerais & Alagoas \\
$22^{\underline{a}}$ & Goiás & São Paulo \\
$23^{\underline{a}}$ & Pernambuco & Minas Gerais \\
$24^{\underline{a}}$ & Piauí & Rio deJ aneiro \\
\hline &
\end{tabular}

Fonte: Elaborado pelos autores.

Além das eficiências, o modelo DEA permite identificar os benchmarks, isto é, os RPPS eficientes que são a referência para o RPPS ineficiente. A tabela 07 mostra o RPPS eficiente que cada RPPS ineficiente deve tomar como referência. Desse modo, os RPPS eficientes de cada modelo têm se próprio como benchmark e os RPPS ineficientes tem seu benchmark mais importante, isto é, mais próximo, segundo o modelo DEA/BCC e o DEA/CCR. 
TABELA 07 - BENCHMARKS DOS RPPS ESTADUAIS.

\begin{tabular}{|c|c|c|}
\hline \multirow{2}{*}{ RPPS } & \multicolumn{2}{|c|}{ BENCHMARKS } \\
\hline & DEA/BCC & DEA/CCR \\
\hline $\mathrm{AC}$ & Acre & Distrito Federal \\
\hline $\mathrm{AL}$ & Alagoas & Amazonas \\
\hline AM & Amazonas & Amazonas \\
\hline BA & Distrito Federal & Distrito Federal \\
\hline $\mathrm{CE}$ & Rio Grande do Norte & Distrito Federal \\
\hline $\mathrm{DF}$ & Distrito Federal & Distrito Federal \\
\hline ES & Distrito Federal & Distrito Federal \\
\hline GO & Distrito Federal & Distrito Federal \\
\hline MA & Maranhão & Amazonas \\
\hline MS & Acre & Distrito Federal \\
\hline MG & Distrito Federal & Distrito Federal \\
\hline PA & Pará & Rondônia \\
\hline PB & Rio Grande do Norte & Distrito Federal \\
\hline PE & Distrito Federal & Distrito Federal \\
\hline PI & Rio Grande do Norte & Distrito Federal \\
\hline RJ & Rio deJaneiro & Amazonas \\
\hline $\mathrm{RN}$ & Rio Grande do Norte & Distrito Federal \\
\hline RS & Distrito Federal & Distrito Federal \\
\hline RO & Rondônia & Rondônia \\
\hline $\mathrm{RR}$ & Roraima & Roraima \\
\hline $\mathrm{SC}$ & Distrito Federal & Distrito Federal \\
\hline $\mathrm{SP}$ & São Paulo & Distrito Federal \\
\hline $\mathrm{SE}$ & Acre & Distrito Federal \\
\hline TO & Tocantins & Tocantins \\
\hline
\end{tabular}

Fonte: Elaborado pelos autores.

Quanto ao modelo DEA/BCC, destacam-se os RPPS de Distrito Federal, o mais eficiente segundo os dois modelos, e o RPPS de Rio Grande do Norte que foram os benchmarks para a maioria dos RPPS ineficientes. Assim como no modelo DEA/BCC, o RPPS de Distrito Federal destacou-se também no modelo DEA/CCR, onde foi benchmark para quinze RPPS ineficientes.

\subsection{ANÁLISE DA EFICIÊNCIA DOS RPPS DAS CAPITAIS ESTADUAIS}

Para identificação dos RPPS de capitais estaduais foi realizado o mesmo processo que identificou a eficiência dos RPPS estaduais. A tabela 08 expõe os inputs: receitas, número de beneficiários e despesas; e outputs: total de benefícios e incremento do patrimônio líquido dos RPPS de capitais estaduais. 
TABELA 08 - INPUTS E OUTPUTS DOS RPPS DE CAPITAIS ESTADUAIS.

\begin{tabular}{cccccc}
\hline DMU & Input1 & Input2 & Input3 & Output1 & Output2 \\
\hline ARA & 293771452 & 3939 & 173000407 & 170650339 & 74418472 \\
BEL & 249470338 & 4454 & 41992437 & 164962629 & 52609980 \\
BH & 757609975 & 14657 & 667018133 & 668257247 & 71407021 \\
BV & 104570384 & 19 & 7719149 & 1976251 & 68953475 \\
CG & 203429820 & 3917 & 227985274 & 197686319 & -72935496 \\
CUI & 155475425 & 2804 & 122511652 & 102570832 & 17032436 \\
CUR & 988979818 & 2491 & 585783534 & 116291417 & 359550375 \\
FLO & 105374611 & 2123 & 104740314 & 95338567 & -13276644 \\
FOR & 466251659 & 12984 & 448353769 & 407136697 & 25042291 \\
GOI & 404967946 & 7170 & 312787116 & 293935380 & 129189168 \\
JP & 152663709 & 4355 & 138651074 & 120615879 & 11473986 \\
MAC & 47569839 & 916 & 32436650 & 28999237 & 10637613 \\
MAN & 326124906 & 5476 & 172436097 & 157894785 & 146041537 \\
NAT & 213826031 & 498 & 144189871 & 147448260 & 79706027 \\
PAL & 97536024 & 398 & 27638291 & 8988728 & 66861187 \\
POA & 1035202971 & 12858 & 873130229 & 803466692 & 166348017 \\
REC & 490470920 & 7871 & 236617019 & 330588957 & 184392265 \\
RB & 61986451 & 117 & 6666086 & 5227362 & 52572204 \\
SAL & 518877751 & 2977 & 509978049 & 502538327 & -9174563 \\
SL & 203847552 & 431 & 166293205 & 46536218 & 42087581 \\
SP & 5276541483 & 79180 & 5312754180 & 5284154587 & -538408 \\
TER & 210212288 & 3922 & 133873578 & 123724025 & 62681710 \\
VIT & 225717687 & 4310 & 3698503 & 175217782 & 48571597 \\
\hline
\end{tabular}

Fonte: Elaborado pelos autores.

A Tabela 09 mostra as eficiências convencional (padrão), invertida e composta normalizada para todos os RPPS de capitais estaduais para os dois modelos (BBC e CCR). 
TABELA 09 - EFICIÊNCIAS DOS RPPS DE CAPITAIS ESTADUAIS, MODELO BCC E CCR.

\begin{tabular}{ccccccc}
\hline \multirow{2}{*}{ DMU } & \multicolumn{3}{c}{ DEA/BCC } & & DEA/CCR \\
& Padrão & Invertida & Composta* & Padrão & Invertida & Composta* \\
\hline ARA & 0,798276 & 0,775952 & 0,671381 & 0,797760 & 0,754010 & 0,683742 \\
BEL & 0,867151 & 0,819587 & 0,687956 & 0,866895 & 0,804517 & 0,695945 \\
BH & 0,961739 & 0,900678 & 0,696820 & 0,961650 & 0,802675 & 0,759224 \\
BV & 1.000 .000 & 1.000 .000 & 0,656720 & 1.000 .000 & 1.000 .000 & 0,655082 \\
CG & 1.000 .000 & 1.000 .000 & 0,656720 & 0,970367 & 1.000 .000 & 0,635670 \\
CUI & 0,784811 & 0,949511 & 0,548558 & 0,754098 & 0,948358 & 0,527826 \\
CUR & 1.000 .000 & 1.000 .000 & 0,656720 & 0,498613 & 1.000 .000 & 0,326633 \\
FLO & 1.000 .000 & 1.000 .000 & 0,656720 & 0,906105 & 1.000 .000 & 0,593573 \\
FOR & 0,925021 & 1.000 .000 & 0,607480 & 0,917934 & 1.000 .000 & 0,601322 \\
GOI & 1.000 .000 & 0,756815 & 0,816424 & 1.000 .000 & 0,699784 & 0,851748 \\
JP & 0,893747 & 1.000 .000 & 0,586942 & 0,852692 & 1.000 .000 & 0,558583 \\
MAC & 1.000 .000 & 1.000 .000 & 0,656720 & 0,798955 & 0,895693 & 0,591711 \\
MAN & 1.000 .000 & 1.000 .000 & 0,656720 & 0,905652 & 1.000 .000 & 0,593276 \\
NAT & 1.000 .000 & 0,477281 & 1.000 .000 & 1.000 .000 & 0,473474 & 1.000 .000 \\
PAL & 1.000 .000 & 1.000 .000 & 0,656720 & 0,828870 & 1.000 .000 & 0,542978 \\
POA & 1.000 .000 & 0,848976 & 0,755900 & 0,909574 & 0,709655 & 0,786046 \\
REC & 1.000 .000 & 1.000 .000 & 0,656720 & 1.000 .000 & 0,681210 & 0,863916 \\
RB & 1.000 .000 & 1.000 .000 & 0,656720 & 1.000 .000 & 0,723485 & 0,836222 \\
SAL & 1.000 .000 & 1.000 .000 & 0,656720 & 1.000 .000 & 0,934176 & 0,698203 \\
SL & 0,487578 & 1.000 .000 & 0,320202 & 0,428696 & 1.000 .000 & 0,280831 \\
SP & 1.000 .000 & 1.000 .000 & 0,656720 & 1.000 .000 & 0,910257 & 0,713871 \\
TER & 0,840854 & 0,900642 & 0,617456 & 0,839351 & 0,882863 & 0,626578 \\
VIT & 1.000 .000 & 0,736982 & 0,829449 & 1.000 .000 & 0,726275 & 0,834395 \\
\hline
\end{tabular}

*Eficiência normalizada.

Fonte: Elaborado pelos autores.

Quanto à eficiência padrão, assim como aconteceu com os RPPS estaduais, o modelo DEA/BCC identificou um maior número de RPPS eficientes comparado ao modelo DEA/CCR. Ao observar a tabela 09 percebe-se que o modelo DEA/BCC identificou quinze RPPS de capitais estaduais eficientes, enquanto que o modelo DEA/ CCR encontrou quinze ineficientes.

Ao observar a tabela 10 percebe-se, mais nitidamente, a inversão dos resultados. O modelo DEA/BCC com quinze RPPS de capitais estaduais eficientes e oito ineficientes e o modelo DEA/CCR oito RPPS de capitais estaduais eficientes e quinze ineficientes. No entanto, todos os oito RPPS eficientes identificados pelo modelo DEA/CC são também eficientes no modelo DEA/BCC. O mesmo ocorre com os RPPS de capitais estaduais ineficientes. 
Na perspectiva da fronteira invertida, o RPPS mais eficiente é aquele que conseguir ter um desempenho mais equilibrado, ou seja, é aquele que conseguir produzir muito de todos os outputs e gastar pouco de todos os inputs, sem se destacar em nenhum especificamente. Nesse caso, a fronteira invertida tenta excluir do grupo de eficientes os RPS que foram considerados eficientes graças ao desempenho muito superior em apenas um único input ou em apenas um único output. Portanto, no modelo DEA/BCC, a fronteira invertida eliminou do grupo de eficientes o RPPS de Boa Vista, Campo Grande, Curitiba, Florianópolis, Macapá, Manaus, Palmas, Recife, Rio Branco, Salvador e São Paulo, restando apenas no grupo de eficientes os RPPS de Goiânia, Natal, Porto Alegre e Vitória. No modelo DEA/CCR apenas o RPPS de Boa Vista apresentou "falsa eficiência".

TABELA 10 - RPPS DE CAPITAIS ESTADUAIS EFICIENTES E INEFICIENTES - EFICIÊNCIA PADRÃO.

\begin{tabular}{cccc}
\hline \multicolumn{2}{c}{ EFICIENTES } & \multicolumn{2}{c}{ INEFICIENTES } \\
\hline DEA/BCC & DEA/CCR & DEA/BCC & DEA/CCR \\
\hline Boa Vista & Boa Vista & Aracaju & Aracaju \\
Campo Grande & Goiânia & Belém & Belém \\
Curitiba & Natal & BH & BH \\
Florianópolis & Recife & Cuiabá & Campo Grande \\
Goiânia & Rio Branco & Fortaleza & Cuiabá \\
Macapá & Salvador & João Pessoa & Curitiba \\
Manaus & São Paulo & São Luís & Florianópolis \\
Natal & Vitória & Teresina & Fortaleza \\
Palmas & & & João Pessoa \\
Porto Alegre & & & Macapá \\
Recife & & & Manaus \\
Rio Branco & & & Palmas \\
Salvador & & & Porto Alegre \\
São Paulo & & & São Luís \\
Vitória & & & Teresina \\
\hline
\end{tabular}

Fonte: Elaborado pelos autores.

O modelo DEA/ BCC identifica os RPPS eficientes tanto com retornos crescentes - quando o aumento da quantidade de inputs determina um aumento mais que proporcional na quantidade de outputs - e constantes de escala - quando o aumento da quantidade de inputs determina um aumento proporcional na quantidade de outputs - enquanto que o modelo DEA/CCR identifica somente os RPPS que foram eficientes com retornos constantes de escala.

Nesse sentido, percebe-se, a partir dos resultados, que os RPPS de Aracaju, Belém, BH, Cuiabá, Fortaleza, João Pessoa, São Luís e Teresina não foram eficientes nem com retornos crescentes e nem retornos constantes de escala. 
Como modelo DEA/BCC também identifica os RPPS eficientes com retornos constantes, logo, os RPPS de Boa Vista, Goiânia, Natal, Recife, Rio Branco, Salvador, São Paulo e Vitória são eficientes com retornos constantes de escala que o modelo DEA/BCCidentificou.

Após identificar quais os RPPS eficientes e ineficientes dos dois modelos DEA/BCC e DEA/CCR - faz-se necessário fazer um ranking e através deste pode-se descobrir qual o RPPS de capital estadual mais eficiente e menos eficiente, para os dois modelos, conforme tabela 11. O ranking é construído a partir dos valores da eficiência normalizada.

TABELA 11 - RANKING DOS RPPS DE CAPITAIS ESTADUAIS - EFICIÊNCIA NORMALIZADA.

\begin{tabular}{cll}
\hline Posição & \multicolumn{1}{c}{ DEA/BCC } & DEA/CCR \\
\hline $1^{\underline{a}}$ & Natal & Natal \\
$2^{\underline{a}}$ & Vitónia & Recife \\
$3^{\underline{a}}$ & Goiânia & Goiânia \\
$4^{\underline{a}}$ & Porto Alegre & Rio Branco \\
$5^{\underline{a}}$ & BH & Vitória \\
$6^{\underline{a}}$ & Belém & Porto Alegre \\
$7^{\underline{a}}$ & Aracaju & BH \\
$8^{\underline{a}}$ & Boa Vista & São Paulo \\
$9^{\underline{a}}$ & Campo Grande & Salvador \\
$10^{\underline{a}}$ & Curitiba & Belém \\
$11^{\underline{a}}$ & Florianópolis & Aracaju \\
$12^{\underline{a}}$ & Macapá & Boa Vista \\
$13^{\underline{a}}$ & Manaus & Campo Grande \\
$14^{\underline{a}}$ & Palmas & Teresina \\
$15^{\underline{a}}$ & Recife & Fortaleza \\
$16^{\underline{a}}$ & Rio Branco & Florianópolis \\
$17^{\underline{a}}$ & Salvador & Manaus \\
$18^{\underline{a}}$ & São Paulo & Macapá \\
$19^{\underline{a}}$ & Teresina & João Pessoa \\
$20^{\underline{a}}$ & Fortaleza & Palmas \\
$21^{\underline{a}}$ & João Pessoa & Cuiabá \\
$22^{\underline{a}}$ & Cuiabá & Curitiba \\
$23^{\underline{a}}$ & São Luís & São Luís \\
\hline
\end{tabular}

Fonte: Elaborado pelos autores.

De acordo com a eficiência normalizada, o RPPS de Natal é o mais eficiente dentre os RPPS de capitais estaduais, os dois modelos o identificaram com a $1^{\underline{a}}$ posição. Além do primeiro lugar no ranking, os dois modelos convergiram também na 3a posição - RPPS de Goiânia - e na última posição, sendo o RPPS 
menos eficiente ou mais ineficiente - RPPS de São Luís. Além das eficiências (padrão, invertida, composta normalizada), o modelo DEA identifica os benchmarks para aqueles RPPS ineficientes. A tabela 12 mostra o benchmark mais importante para cada RPPS de capitais estaduais.

TABELA 12 - BENCHMARKS DOS RPPS DE CAPITAIS ESTADUAIS.

\begin{tabular}{lll}
\hline \multirow{2}{*}{ RPPS } & \multicolumn{1}{c}{ BENCHMARKS } \\
\cline { 2 - 3 } Aracaju & Natal & \multicolumn{1}{c}{ DEA/CCR } \\
Belém & Vitória & Natal \\
BH & Natal & Natal \\
Boa Vista & Boa Vista & Boa Vista \\
Campo Grande & Campo Grande & São Paulo \\
Cuiabá & Campo Grande & Natal \\
Curitiba & Curitiba & Rio Branco \\
Florianópolis & Florianópolis & São Paulo \\
Fortaleza & Campo Grande & Natal \\
Goiânia & Goiânia & Goiânia \\
João Pessoa & Campo Grande & Natal \\
Macapá & Macapá & Natal \\
Manaus & Manaus & Rio Branco \\
Natal & Natal & Natal \\
Palmas & Palmas & Rio Branco \\
Porto Alegre & Porto Alegre & Natal \\
Recife & Recife & Recife \\
Rio Branco & Rio Branco & Rio Branco \\
Salvador & Salvador & Salvador \\
São Luís & Natal & Natal \\
São Paulo & São Paulo & São Paulo \\
Teresina & Natal & Natal \\
Vitória & Vitória & Vitória \\
\hline
\end{tabular}

Fonte: Elaborado pelos autores.

O RPPS de Natal, o mais eficiente segundo a eficiência normalizada dos dois modelos utilizados, destacou-se por ser o benchmark de quatro RPPS ineficientes no modelo DEA/BCC e de dez RPPS ineficientes no modelo DEA/CCR. Destaca-se, ainda, o RPPS de Campo Grande que foi benchmark de três RPPS ineficientes no modelo DEA/BCC e o RPPS de Rio Branco que foi referência para três RPPS ineficientes também, no modelo DEA/CCR. 


\section{CONSIDERAÇÕES FINAIS}

No presente estudo foi utilizada a Análise Envoltória de Dados (DEA) para a avaliação dos maiores RPPS brasileiros no ano de 2014. O objetivo do estudo de identificar os RPPS mais eficientes em 2014 foi atingido através da eficiência padrão, mas foi a aplicação da fronteira invertida que permitiu identificar também os RPPS com "falsa eficiência". Além disso, a fronteira composta normalizada (obtida a partir da eficiência padrão e da eficiência invertida) permitiu a identificação do RPPS com melhor e pior desempenho. Primeiramente, identificou os RPPS estaduais mais eficientes através dos modelos DEA/ BCC e DEA/CCR e logo após a avaliação foi realizada para os RPPS de capitais estaduais.

Diante dos resultados do estudo, percebe-se que a fronteira invertida identificou mais RPPS com "falsa eficiência" no modelo DEA/BCC. No modelo DEA/CCR identificou apenas um RPPS estadual e outro de capital estadual. Esse resultado pode ser explicado porque o modelo DEA/BCC considera os três retornos de escala, enquanto o DEA/CCR considera apenas o retorno constante de escala. Percebe-se ainda que o RPPS estadual mais eficiente - Distrito Federal - e o RPPS de capital estadual mais eficiente - Natal - não apresentaram falsa eficiência, pelo contrário, em ambos os modelos, os dois RPPS em questão apresentaram o menor score de eficiência na fronteira invertida.

Mesmo diante dos resultados desse estudo, cabe destacar que ainda existe um grande potencial de pesquisa no que se refere ao desempenho dos RPPS, já que não existem muitos estudos com esse enfoque. Grande parte da avaliação dos RPPS baseia-se na redução da relação entre beneficiário/contribuinte, sem considerar como os RPPS são administrados.

O diagnóstico realizado por este estudo foi fundamental para identificar os RPPS estaduais e de capitais estaduais eficientes, bem como, os RPPS com falsa eficiência, os benchmarks dos RPPS ineficientes e a construção do ranking dos mais eficientes. Ressalta-se que este trabalho pode ser estendido para a análise dos determinantes, isto é, os motivos que tornaram determinados RPPS mais ou menos eficientes no período analisado.

\section{REFERÊNCIAS}

ALI, Agha Iqbal. (1993). Streamlined computation for data envelopment analysis. European J ournal of Operational Research, v. 64, p. 61-67.

ANGULO MEZA, L., BIONDI NETO, L., SOARES DE MELLO, J.C.C.B. and GOMES, E.G. (2005) ISYDS - Integrated System for Decision Support (SIAD Sistema Integrado de Apoio à Decisão): A Software Package for Data Envelopment Analysis Model, Pesquisa Operacional, Vol 25, No 3, pp. 493-503. 
BALERA, Wagner. (2003). Sistema de Seguridade Social. 3. ed. São Paulo: Ed. dos Tribunais.

BANKER, R.D.; CHARNES, A.; COOPER, W.W. (1984). Some models for estimating technical scale inefficiencies in data envelopment analysis. Management Science, v. 30, n. 9, p. 1078-1092.

BOSCHETTI, Ivanete. (2003). Implicações da Reforma da Previdência na Seguridade Social brasileira. In: Psicol. soc. v.15, n.1, p. 57-96, jan. /jun. [S.l].

BRASIL. Constituição (1988). Constituição da República Federativa do Brasil: promulgada em 5 de outubro de 1988. Organização do texto: J uarez de Oliveira. 4. ed. São Paulo: Saraiva, 1990.

. Lei n. 6.435, de 15 de julho de 1977. Dispõe a criação da Previdência Complementar Privada.

. Lei n. 9.717, de 27 de novembro de 1998. Dispõe sobre os Regimes Próprios de Previdência Social dos Servidores Públicos da União, dos Estados, do Distrito Federal e dos Municípios e os militares dos Estados e do Distrito Federal.

COOPER, W., SEIFORD, L. e TONE, K. (2000). Data Envelopment Analysis: a comprehensive text with models, applications, references and DEA-Solver software, Massachusetts. USA: Kluwer Academic Publishers.

ENTANI, T.; MAEDA, Y.; TANAKA, H. (2002). Dual models of interval DEA and its extensions tointerval data. European J ournal of Operational Research, v. 136, p. 32-45.

FOLLADOR, Renato. O Grande Propagandista Da Previdência Privada é o INSS. Depoimento. [Janeiro/fevereiro 2014]. Paraná: Fundo de Pensão. Entrevista concedida a René Ruschel.

IBRAHIM, Fábio Zambitte. (2005). in Resumo de Direito Previdenciário, 4. ed. Rio de J aneiro: Editora Ímpetus.

GIAMBIAGI. Fábio. Crise na previdência social. Entrevista. [9 de março de 2014]. Rio de J aneiro: O Dia. Entrevista concedida a Alexandra Couto. 
Leta, F.R.; Soares de Mello, J.C.C.B.; Gomes, E.G.; Angulo Meza, L. (2005). Métodos de melhora de ordenação em DEA aplicados à avaliação estática de tornos mecânicos. Investigação Operacional, v. 25, n. 2.

LEWGOY, Henrique. (2008). Financiamento e Gestão Pública dos Regimes Próprios de Previdência Social dos Municípios do Rio Grande do Sul. Curso de Especialização (Gestão Pública e Controle Externo) - Escola Superior de Gestão de Controle Francisco J uruena, Tribunal de Contas do Estado, Rio Grande do Sul.

LINS, M.P.E.; ANGULO-MEZA, L. (2000). Análise Envoltória de Dados e perspectivas de integração no ambiente de Apoio à Decisão. Rio de Janeiro: Editora da COPPE/ UFRJ .

NOVAES, L. F. L. (2002). Envoltória Sob Dupla ótica aplicada na avaliação imobiliária em ambiente do sistema de informação geográfica. Tese (Doutorado), Universidade Federal do Rio de J aneiro - UFRJ , Rio de J aneiro.

SCHERMAN, K. G. Introdução. In: THOMPSON, Lawrence. (2000). Mais velha e mais sábia: a economia dos sistemas previdenciários. Tradução de Celso Barroso leite. Brasília: MPAS. (Coleção Previdência Social. Série Traduções, v. $4)$.

SOARES DE MELLO, J .C.C.B.; ANGULO MEZA, L.; GOMES, E.G.; SERAPIÃO, B.P.; LINS, M.P.E. (2003). Análise de Envoltória de Dados no estudo da eficiência e dos benchmarks para companhias aéreas brasileiras. Pesquisa Operacional, v. 23, n. 2, p. 325-345.

Yamada, Y., Matui, T. and Sugiyama, M. (1994) New analysis of efficiency based on DEA, J ournal of the Operations Research Society of J apan, Vol 37, No 2, pp. 158-167. 